\title{
Weitere Versuche über die zellfreie Gärung.
}

\author{
von \\ Eduard Buchner und Wilhelm Antoni.
}

(Aus dem chemischen Laboratorium der landwirtschaftlichen Hochschule zu Berlin.)

(Der Redaktion zugegangen am 15. März 1905.)

\section{Einfluß von Sauerstoff auf die Zymase. $\left.{ }^{1}\right)$}

Hefepreßsaft büßt beim Stehen an der Luft innerhalb weniger Tage seine Gärkraft ein. Ehe erkannt war, daß es sich dabei um eine Zerstörung der Zymase durch die proteolytischen Enzyme des Preßsaftes handelt, konnte man vermuten, daß ein schädlicher Einfluß des Sauerstoffs der Luft daran Schuld trägt. ${ }^{2}$ ) Auch E. Duclaux ${ }^{3}$ ) und P. Mazé ${ }^{4}$ ) äußern ähnliche Annahmen. Zwar zeigte sich bald, daß es für das Zurückgehen der Gärkraft des Preßsaftes gleichgültig ist, ob er in vollgefüllten verstopften Flaschen oder in offenen Gefäßen aufbewahrt wird. ${ }^{5}$ ) Trotzdem schien es wünschenswert, mittels vergleichender Versuche unter Einleiten von Sauerstoff bezw. Wasserstoff die Frage genauer zu prüfen. Dabei war ein wesentlicher Unterschied nicht nachzuweisen. ${ }^{6}$ )

1) Wie Buchner und Meisenheimer vor kurzem gezeigt haben (Berichte d. Deutsch. chem. Ges., Bd. XXXVIII, S. 621, 1905), ist die Gärwirkung der Hefe auf die Anwesenheit zweier verschiedener Enzyme zurückzuführen, deren eines den Zucker in Milchsäure, deren anderes die Milchsäure in Alkohol und Kohlendioxyd spaltet. Da es für die folgenden Betrachtungen nicht möglich ist, diese beiden Gärungsenzyme auseinanderzuhalten, seien sie unter dem auch früher gebräuchlichen Namen ‘Zymase zusammengefaßt.

$\left.{ }^{2}\right)$ Buchner, Ber. d. Deutsch. chem. Ges., Bd. XXX, S. 119 (1897).

3) Microbiologie, Paris, Bd. III, S. 181 (1900).

4) Compt. rend., Bd. CXXXVIII, S. 1515 (1904).

5) E. und H. Buchner und M. Hahn, Die Zymasegärung, S. 97, München 1903.

6) $\mathrm{Zu}$ demselben Ergebnis ist kürzlich bei Arbeiten mit käuflicher Acetondauerhefe (sog. Zymin) Frl. O. Grigoriew gekommen (Diese Zeitschrift, Bd. XLII, S. 316, 1904). 
Zunächst wurde in zwei mit Preßsaft aus frischer Bierunterhefe $^{1}$ ) halbvoll gefüllte Flaschen $1 / 4$ Stunde lang Sauerstoff bezw. Wasserstoff eingeleitet. Der Wasserstoff war durch Permanganatlösung gereinigt worden. Die sorgfältig verschlossenen Gefäße wurden dann 24 Stunden in Eiswasser aufbewahrt und hierauf die Gärkraft in gewöhnlicher Weise ${ }^{2}$ ) bestimmt. Nach Beendigung der Gärung wurde die im Luftraum der Kölbchen angesammelte Kohlensäure noch durch Luft verdrängt.

\section{Tabelle I.}

Gärkraft des Preßsaftes nach Stehen mit Sauerstoff bezw. Wasserstoff.

Je $20 \mathrm{ccm}$ Preßsaft $+8 \mathrm{~g}$ Rohrzucker $+0,2 \mathrm{ccm}$ Toluol. $22^{\circ}$.

\begin{tabular}{c|c|c|c|c|c}
\hline \multirow{2}{*}{$\begin{array}{c}\text { 24 Stunden } \\
\text { gestanden } \\
\text { mit }\end{array}$} & \multicolumn{3}{|c|}{ Kohlendioxyd in Grammen nach Stunden } \\
\cline { 2 - 6 } & 24 & 48 & 72 & \multicolumn{2}{|c}{$\begin{array}{c}\text { Luftdurchleitung } \\
\text { ohne }\end{array}$} \\
\hline \multirow{2}{*}{ Sauerstoff } & 0,89 & 0,98 & 0,99 & 1,01 & 1,04 \\
& 0,89 & 0,97 & 0,99 & 1,01 & 1,03 \\
& 0,92 & 1,01 & 1,03 & 1,05 & 1,08 \\
Wasserstoff & 0,90 & 0,99 & 1,02 & 1,05 & 1,08
\end{tabular}

Die zwischen den Versuchen mit Sauerstoff- bezw. Wasserstoffeinwirkung hervortretenden Unterschiede sind so gering - sie betragen nur ungefähr $4 \%$ der Gärwirkung -, daß sie innerhalb der Fehlergrenzen liegen, wie durch folgende maßgebendere Versuche mit ständiger Durchleitung der beiden Gase während 24stündigen Verlaufes der Gärung bewiesen wird.

Die Anordnung dieser Versuche (Tab. II) machte insofern einige Schwierigkeiten, als beim Einleiten der Gase in den Preßsaft ungeheures

1) $\mathrm{Zu}$ diesen und den folgenden Versuchen stand uns ausgezeichnete Hefe aus der Brauerei von Julius Bötzow, Berlin, zur Verfügung, wofür wir auch an dieser Stelle besten Dank zum Ausdruck bringen.

) Vergl. «Die Zymasegärung», S. 80, 1903. 
Schäumen eintrat. Wir waren daher gezwungen, jedes Versuchskölbchen $(150 \mathrm{ccm}$ Inhalt, beschickt mit $40 \mathrm{ccm}$ Preßsaft, $16 \mathrm{~g}$ Rohrzucker und 0,02-0,03 g Thymol), mit einem zweiten, leeren Kölbchen zu verbinden, in welchem der übergehende Schaum sich ohne Schädigung ablagern konnte. Erst dies zweite Kölbchen trug dann den Meisslschen Schwefelsäuregärverschluß, an welchen sich vorsichtshalber noch ein Chlorcalciumrohr anschloß. Gärkölbchen, leere Vorlage und Chlorcalciumrohr wurden vor und nach dem Versuche sowohl mit den Gasen gefüllt wie auch nach halbstündigem Durchleiten von Luft gewogen. Die hierbei auftretende Differenz ist bei allen Angaben berücksichtigt. Versuchstemperatur war $22^{\circ}$; Sauerstoff und Wasserstoff wurden vor dem Einleiten mit Schwefelsäure und Chlorcalcium getrocknet, der Wasserstoff durch Permanganatlösung gereinigt. Zum Vergleiche kam auch die Gärkraft des Saftes in der gewöhnlichen Weise ohne Durchleiten von Gas zur Bestimmung. Die geringere Gärkraft in letzterem Falle rührt jedenfalls zum größten Teil von der Übersättigung des Saftes mit Kohlendioxyd her; wahrscheinlich hat außerdem auch die Kohlensäure eine geringe schädigende Wirkung auf die Zymase.

Tabelle II.

Gärkraft bei Sauerstoff-, bezw. Wasserstoff-und ohne Gas-Einleitung.

Je $40 \mathrm{ccm}$ Preßsaft $+16 \mathrm{~g}$ Rohrzucker $+0,02 \mathrm{~g}$ Thymol. $22^{\circ}$.

\begin{tabular}{|c|c|c|c|}
\hline \multirow[t]{2}{*}{ Datum } & \multirow[t]{2}{*}{ Durchgeleitetes Gas } & \multicolumn{2}{|c|}{$\begin{array}{c}\text { Kohlendioxyd } \\
\text { in Grammen nach Stunden }\end{array}$} \\
\hline & & 12 & 24 \\
\hline 25. I. 05 & $\begin{array}{l}\text { Sauerstoff . . . . } \\
\text { Wasserstoff . . . . } \\
\text { Ohne Gasdurchleitung }{ }^{1} \text { ). }\end{array}$ & $\begin{array}{l}1,97 \\
2,08 \\
1,54\end{array}$ & $\begin{array}{l}3,62 \\
3,59 \\
3,20\end{array}$ \\
\hline 31. I. 05 & $\begin{array}{l}\text { Sauerstoff . . . . } \\
\text { Wasserstoff . . . . } \\
\text { Ohne Gasdurchleitung . . }\end{array}$ & $\begin{array}{l}1,85 \\
1,90 \\
1,50\end{array}$ & $\begin{array}{l}2,77 \\
2,85 \\
2,55\end{array}$ \\
\hline
\end{tabular}

1) Die Gesamtgärkraft des Preßsaftes betrug in beiden Fällen etwa $4 \mathrm{~g} \mathrm{CO}_{2}$ für $40 \mathrm{ccm}$, die Reaktion ist nach 24 Stunden durchaus nicht zu Ende. 
Versuche zur Trennung von Zymase und Invertase.

Preßsaft aus untergäriger Bierhefe vergärt Rohrzucker ebenso rasch wie Traubenzucker. Der Vergärung der Disaccharide durch lebende Hefe geht, soweit bis jetzt feststeht, eine Spaltung in Hexosen durch Enzyme voraus. Es schien nun wünschenswert, diese Annahme am Preßsaft $\mathrm{zu}$ prüfen und die Trennung der Invertase und der Zymase zu versuchen, eine Aufgabe, deren Durchführung voraussichtlich wegen der ähnlichen Eigenschaften jener Stoffe und der Veränderlichkeit der Zymase allerdings auf große Schwierigkeiten stoßen mußte. Leider ist es bis jetzt auch nicht gelungen, trotz verschiedenster Versuchsanordnungen, zu einem positiven Ergebnis zu kommen.

Da nach früheren Versuchen Zymase nicht zu dialysieren vermag, wogegen getrocknete Hefe (nicht aber vollkommen gesunde, frische) Invertase in die umgebende Flüssigkeit diffundieren läßt, wurde zunächst probiert, die Invertase aus AcetonDauerhefe durch verdünnte Glyzerinlösungen herauszuziehen. Durch die unverletzten Zellmembranen zurückgehalten, sollte dabei die Zymase im Innern der Zellen verbleiben; und nach Entfernen der Invertase erst durch Zerreißen der Zellmembranen in Lösung gebracht werden. ${ }^{1}$ ) Es hat sich aber gezeigt, daß aus Acetondauerhefe durch 10\% ige Glyzerinlösungen zwar recht wirksame Extrakte ausgezogen werden können, welche viel Rohrzucker invertieren; aber eine Erschöpfung der Acetondauerhefe an Invertase ist selbst nach 4tägigem Schütteln mit der 10 fachen Menge 10\% iger Glyzerinlösung unter täglicher Erneuerung der Extraktionsflüssigkeit nicht zu erreichen. Nach Isolierung der zurückbleibenden Acetondauerhefe durch $\mathrm{Ab}$ saugen erwies sich jedoch beim Übergießen mit Zuckerlösung, daß alle Gärkraft bereits verschwunden war. Das Durchtreten der Invertase durch die unverletzte Zellmembran dauert demnach viel zu lange, als daß auf diesem Wege eine Trennung von der Zymase ausführbar wäre.

I) Vergl. R. und W. Albert, Zentralbl. f. Bakteriologie II, Bd. VII, S. 742 (1901).

Hoppe-Seyler's Zeitschrift f. physiol. Chemie. XLIV. 
Ein rascheres Diffundieren der Invertase konnte erwartet werden, wenn es nicht durch die Zellmembran, sondern durch grobporiges Pergamentpapier vor sich gehen sollte; ein Diffundieren der Zymase war nach früheren Ergebnissen auch dabei nicht zu befürchten. ${ }^{1}$ ) Wir benützten $\mathrm{zu}$ unseren Versuchen den Gürberschen Dialysierapparat, welcher gestattet, die zu dialysierende Flüssigkeit in einem Pergamentpapierschlauch durch einen auf- und niedergehenden Rührer in fortwährender Bewegung $\mathrm{zu}$ erhalten. Die Dialyse fand gegen eine Salzlösung statt, die in $1 \mathrm{l} 1 \mathrm{~g}$ sekundäres Kaliumphosphat, 0,4 g Kochsalz, 0,4 g kristallisiertes Magnesiumsulfat und 0,2 g Gips enthielt. Der Versuch wurde 2 mal 24 Stunden unter Wechseln der äußeren Lösung nach dem ersten Tage in Gang gelassen und dabei die Temperatur durch Einsenken in Eiswasser möglichst niedrig gehalten. Bei der Prüfung mit Rohrzuckerlösung zeigte das nach 48 Stunden erhaltene Diffusat noch starke invertierende Kraft, ebenso aber auch der Preßsaft im Innern des Pergamentschlauches nach 2 tägiger Dialyse, wogegen er seine Gärwirkung auf Zucker bis auf geringe Spuren eingebüßt hatte. $20 \mathrm{ccm}$ gaben auf Zusatz von $8 \mathrm{~g}$ Zucker nunmehr nur noch $0,03 \mathrm{~g} \mathrm{CO}_{2}$ ! Die Diffusion der Invertase findet demnach auch durch Pergamentpapier selbst im Gürberschen Apparat viel zu langsam statt. Dieses Resultat, das sich bei mehreren analogen Versuchen gleich blieb, stimmt überein mit den inzwischen bekannt gewordenen Versuchen aus Hüfners Laboratorium von $\mathrm{B}$. $\mathrm{Hafner},{ }^{2}$ ) wonach bei 8 tägigem Dialysieren einer Invertaselösung unter anhaltender Bewegung des Apparates weitaus der größte Teil des Enzyms in der Innenflüssigkeit geblieben war, während das Diffusat nur eine verhältnismäßig geringe Menge davon enthielt.

Nachdem die beschriebenen Dialysierversuche nicht zum Ziele geführt hatten, schien eine Trennung der Invertase und Zymase noch möglich durch fraktionierte Fällung mit Alkohol.

1) Buchner und Rapp, Ber. d. Deutsch. chem. Ges., Bd. XXXI, S. 214 (1898).

2) Diese Zeitschrift, Bd. XLII, S. 1 (1904). 
Nach den Angaben von O'Sullivan und Tompson') wird Invertase aus wässerigen Lösungen durch Zusatz von Äthylalkohol bis zu 47\% bis auf geringe Spuren ausgefällt, wogegen die Abscheidung der Zymase viel größere Mengen Alkohol erfordert; falls eine vollständige Fällung dieses Enzymes beabsichtigt ist, müssen jedenfalls mehr als 4 Vol. Alkohol von $96 \%$, wahrscheinlich aber sogar 12 Vol. davon verwendet werden. ${ }^{2}$ )

$50 \mathrm{ccm}$ von sehr gärkräftigem Hefepreßsaft wurden unter Turbinieren und Eiskühlung in $45 \mathrm{ccm}$ absoluten Alkohol einfließen gelassen und, da die flockige Beschaffenheit des reichlichen Niederschlages ein Absaugen ausschloB, in einer Zentrifuge mit 3000 Umdrehungen in der Minute 5 Minuten lang zentrifugiert. Die überstehende Flüssigkeit wurde nunmehr von der entstandenen Fällung I abgegossen, letztere zweimal mit je $50 \mathrm{ccm}$ absolutem Alkohol angerührt und wieder abzentrifugiert, sodann auf einer Nutsche abgesaugt, zweimal mit $50 \mathrm{ccm}$ absolutem Alkohol und endlich mit $50 \mathrm{ccm}$ Äther gewaschen. Der Niederschlag. I, im Vacuum über Schwefelsäure getrocknet, stellt ein rein weißes Pulver vor, das in Wasser nur mehr zum Teil löslich ist. Ausbeute: $5 \mathrm{~g}$. Vom ersten Eingießen des Preßsaftes in den Alkohol bis zum Einstellen des Präparates ins Vacuum waren 25 Minuten verstrichen.

Eine Fällung II erhält man durch Eintragen der von Fällung I abgegossenen Flüssigkeit in viel Alkohol und Äther. Um die Substanzen nur möglichst kurz mit den Fällungsmitteln in Berührung zu lassen, wurden von neuem $180 \mathrm{ccm}$ desselben Preßsaftes, der zur Herstellung der Fällung I gedient hatte, mit $160 \mathrm{ccm}$ absolutem Alkohol gemischt und das Ganze wie bei Herstellung der Fällung I zentrifugiert. Der am Boden sitzende Niederschlag wurde sodann beseitigt, die überstehende Flüssigkeit aber unter Umschütteln abermals eingetragen in $1300 \mathrm{ccm}$ absoluten Alkohol $+1400 \mathrm{ccm}$ Äther. Die so erzielte Fällung II, von der Flüssigkeit durch Abgießen und Zentrifugieren befreit, zweimal mit je $50 \mathrm{ccm}$ Alkohol angerührt und abzentrifugiert, dann auf der Nutsche noch zweimal mit den gleichen Mengen absoluten Alkohols und hierauf mit $50 \mathrm{ccm}$ Äther gewaschen, wurde im Vacuum über Schwefelsäure getrocknet, schließlich als ein weißes Pulver erhalten, das in Wasser leicht löslich ist. Ausbeute: $3 \mathrm{~g}$. Auch hier hatte die ganze Herstellung aus dem Preßsaft 25 Minuten in Anspruch genommen.

Zur Gärkraftbestimmung wurde je $1 \mathrm{~g}$ der Fällungen mit $10 \mathrm{ccm}$ Wasser angerührt und unter Zusatz von $1 \mathrm{~g}$ Glukose und $0,1 \mathrm{ccm}$ Toluol in einem einseitig geschlossenen U-Röhrchen

1) Journal of the Chem. Soc., Bd. LVII, S. 928 (1890).

2) E. u. H. Buchner u. M. Hahn, Zymasegärung, S. 236, 237 (1903). 
bei $30^{\circ}$ aufgestellt. Fällung I gab nach zwei Tagen überhaupt noch keine Gasentwicklung, nach 5 Tagen $3 \mathrm{ccm}$ Gas, eine Menge, die bei weiterem Stehen nicht mehr zunahm; Fällung II lieferte dabei nach 2 Tagen $4 \mathrm{ccm}$ Gas, nach 3 Tagen $4,2 \mathrm{ccm}$, womit die Gasentwicklung beendet war. In beiden Fällen ließ sich demnach nur eine äußerst geringe Gärwirkung nachweisen. Während also die hier angewandten Gesamtmengen von Alkohol und Äther auf einmal zugesetzt im allgemeinen genügen, aus dem Preßsaft die ganze wirksame Substanz unter vollständiger Erhaltung der Gärkraft als pulverigen Niederschlag auszufällen, ${ }^{1}$ ) konnte bei vorliegendem Versuch weder in der I. noch in der II. Fällung nennenswerte Gärkraft nachgewiesen werden. Die Zymase war also entweder infolge der verzögerten Arbeitsweise und der längeren Berührung mit wasserhaltigem Alhohol zerstört worden oder die Fällung derselben durch Alkohol und Äther beruht mehr auf einem Mitreißen beim Niederfallen der großen Mengen von Eiweißkörpern, das bei der fraktionierten Fällung nicht eintritt, weil die Hauptmenge der Eiweißkörper schon bei der I. Fällung niedergeschlagen wird, durch so wenig Alkohol, daß ein Niederreißen der Zymase noch nicht möglich ist.

In Fällung II ließen sich außerdem noch beträchtliche Mengen von Invertase nachweisen. Dazu wurde $1 \mathrm{~g}$ Niederschlag in $50 \mathrm{ccm}$ einer $13 \%$ igen Rohrzuckerlösung aufgelöst. Die Anfangsdrehung betrug $9^{0} 15^{\prime}$; nach 24stündigem Digerieren bei $30^{\circ}$ war sie auf $-1^{0}$ zurückgegangen. Dieser Versuch beweist also, daß noch eine erhebliche Menge Invertase vorhanden war.

Nachdem somit alle Bemühungen zur Trennung der Zymase von der Invertase gescheitert sind, bleibt nur noch die Möglichkeit, einen Organismus zur Herstellung des Preßsaftes heranzuziehen, welcher von vornherein keine Invertase enthält, um auf diese Weise die Zymasewirkung bei Abwesenheit von Invertase zu untersuchen. Herr R. Gaunt hat es übernommen, derartige Arbeiten mit Saccharomyces apiculatus auszuführen.

1) «Die Zymasegärung*, S. 237 (1903). 


\section{Invertase und Zymase bei hohen Zucker- konzentrationen.}

Nach Th. Bokorny ${ }^{1}$ ) wird die Invertase bei gewöhnlicher Temperatur durch hochkonzentrierte Zuckerlösungen beträchtlich stärker gehemmt als die Zymase, sodaß sehr konzentrierte Traubenzuckerlösungen durch Hefe zwar noch vergoren werden, nicht aber ebenso hochprozentige Rohrzuckerlösungen, weil angeblich die hydrolytische Wirkung der Invertase und Spaltung der Saccharose nicht mehr eintritt. Diese merkwürdigen Angaben, welche bereits in die Literatur übergegangen sind, ${ }^{2}$ ) schienen uns der Bestätigung dringend bedürftig. Bei Bokornys Versuchsanordnung mußte, damit die Enzyme mit dem Zucker in Reaktion treten konnten, die Trennung der beiden Stoffe durch die Zellmembran und den Plasmaschlauch erst überwunden werden. Viel richtiger wäre es daher gewesen, solche Versuche mit Hefepreßsaft anzustellen. Ferner ist es wegen der Löslichkeitsverhältnisse der untersuchten Kohlenhydrate (es lösen sich bei $15^{0}$ nur etwa $44^{\circ} ; 0$ Traubenzucker, ${ }^{3}$ ) während von Rohrzucker bei $15^{\circ} 66 \%$ in Lösung gehen, $\left.{ }^{4}\right)$ ) überhaupt unmöglich, so hochprozentige (z. B. 59 bezw. 74\%) Traubenbezw. Rohrzuckerlösungen herzustellen, wie sie der Autor durch Mischen der kristallisierten Zucker mit bis zu einem Wassergehalt von $70 \%$ abgepreßter Hefe erhalten haben will. Das heißt, die Zucker werden zum Teil ungelöst geblieben sein, was in höherem Grade für den schwerer löslichen Traubenzucker zutreffen wird.

Die Resultate von Bokorny, soweit es sich um die höher konzentrierten Lösungen handelt, bei denen die fraglichen Unterschiede erst auftreten, sind also nicht mit Lösungen von gleicher Konzentration erhalten, sondern mit hochprozentigen'Rohrzucker- und weniger konzentrierten Glukoselösungen, und können

1) Chem.-Ztg., Cöthen, Bd. XXVII, S. 1106 (1903).

2) E. v. Lippmann, Chemie der Zuckerarten, Bd. II, S. 1892 (1904).

3) Vergl. E. v. Lippmann, Chemie der Zuckerarten, Bd. I, S. 266 (1904).

4) Vergl. ebenda, Bd. II, S. 1088. 
daher überhaupt nicht verglichen werden. Dazu kommt noch, daß nach den in der Literatur vorliegenden Angaben Traubenzuckerlösungen nicht fadenziehend und weniger zähflüssig sind als gleichprozentige Rohrzuckerlösungen, ${ }^{1}$ ) was für die Geschwindigkeit von Enzymwirkungen von Einfluß sein dürfte.

Wir haben nun, soweit es die Löslichkeitsverhältnisse der beiden Zucker zulassen, in Preßsaft möglichst viel davon aufgelöst, aber bei gleicher Konzentration beider Zucker keine wesentlichen Unterschiede zwischen Rohr- und Traubenzucker konstatieren können (Versuch I und II). Nimmt man dagegen solche Mengen, daß zwar der Rohrzucker, jedoch nicht der Traubenzucker ganz in Lösung geht, dann allerdings tritt, wie Bokorny beobachtet hat, mit Traubenzucker die Gärung rascher ein (Versuch III). - Das entwickelte Kohlendioxyd wurde volumetrisch bestimmt, in einem dem Hayduckschen ${ }^{2}$ ) ähnlichen, aber viel kleineren und mit Quecksilber als Sperrflüssigkeit beschickten Apparat.

I. In je $10 \mathrm{ccm}$ frischen Preßsaft wurden $5 \mathrm{~g}$ feingepulverter Trauben- bezw. Rohrzucker eingetragen. Bei $22^{\circ}$ entwickelten sich nach 45 Minuten in beiden Fällen $20 \mathrm{ccm} \mathrm{CO}_{2}$. Die Kölbchen waren in Wasser getaucht, um die Temperatur gleichmäßig zu halten. Übersättigungserscheinungen wurden durch häufiges Schütteln der Kölbchen unter Druckverminderung durch Senken des Quecksilbergefäßes verhindert. Bei dieser ungefähr $33 \%$ igen Lösung war-also in Übereinstimmung mit Bokorny ein Unterschied zwischen beiden Kohlenhydraten nicht zu bemerken. Die beiden. Zucker gingen im Laufe des Versuches vollkommen in Lösung.

II. In je $10 \mathrm{ccm}$ Preßsaft wurden $8 \mathrm{~g}$ Trauben- bezw. Rohrzucker eingetragen und bei $22^{\circ}$ aufgestellt. Nach $2^{1 / 8}$ Stunden war beim Versuche mit Traubenzucker noch keine vollständige Lösung eingetreten und $12 \mathrm{ccm} \mathrm{CO} \mathrm{C}_{2}$ entwickelt. Der Rohrzucker hatte sich dagegen bald nach Beginn des Versuches gelöst und $10 \mathrm{ccm} \mathrm{CO}_{2}$ geliefert. Der Traubenzucker war auch nach 24 Stunden noch nicht ganz gelöst; es dauert demnach sehr lange, bis sich die mögliche Höchstkonzentration von etwa $44 \%$ herstellt.

III. In je $10 \mathrm{ccm}$ Preßsaft wurden $10 \mathrm{~g}$ feingepulverter Trauben(1904).

1) Vergl. E. v. Lippmann, Chemie der Zuckerarten, Bd. I, S. 266

2) Vergl. P. Lindner, Mikroskopische Betriebskontrolle, S. 263 (Berlin 1905). 
bezw. Rohrzucker eingetragen. Nach $1 \frac{1}{1}$ Stunden bei $22^{\circ}$ und häufigem Schütteln war der Rohrzucker bis auf Spuren gelöst, vom Traubenzucker mochte schätzungsweise ungefähr die Hälfte ungelöst geblieben sein. Entwickelt waren bei Traubenzucker $20 \mathrm{ccm} \mathrm{CO}_{8}$, bei Rohrzucker $6 \mathrm{ccm}$. Da aber ersterer überhaupt nur zum Teil in Lösung gegangen war, sind die Versuche bei verschiedenen Zuckerkonzentrationen ausgeführt worden und haben keine Beweiskraft in dem von Bokorny gewollten Sinne.

Schließlich haben wir noch versucht, ob in konzentrierten Rohrzuckerlösungen nach Zusatz von Preßsaft Invertierung nachzuweisen ist. In $10 \mathrm{ccm}$ Wasser wurden $20 \mathrm{~g}$ Rohrzucker gelöst und sorgfältig $0,2 \mathrm{ccm}$ frischer Hefepreßsaft darunter gemischt. Das Drehvermögen der Lösung betrug nunmehr $57^{\circ}$ 15 $^{\prime}$. Nach 5 Stunden Stehen bei Zimmertemperatur war es auf $55^{\circ}$, nach 24 Stunden auf $46^{\circ}$ zurückgegangen, womit die Wirksamkeit der Invertase auch in dieser etwa $66 \%$ igen Rohrzuckerlösung bewiesen ist.

Diese Ergebnisse stimmen überein mit Beobachtungen Hayducks, ${ }^{1}$ ) welcher in $70 \%$ iger Saccharoselösung Gärung festgestellt und den gebildeten Alkohol bestimmt hat. Es müssen demnach in dieser hoch konzentrierten Lösung sowohl die Invertase, wie die Zymase ihre Wirksamkeit entfaltet haben.

\section{Gärkraft von zerriebener Acetondauerhefe.}

Damit die Gärwirkung von Dauerhefe auf Zuckerlösungen zustande kommen kann, muß erst Flüssigkeit in das Innere der Zellen hineindiffundieren, dort die Zymase in Lösung überführen und mit gleichfalts durch Diosmose in die Zellen gelangtem Kohlenhydrat in Berührung bringen. Man könnte demnach vermuten, daß von der Zellmembran befreite, zerriebene Dauerhefe viel schnellere Zerlegung einer Zuckerlösung bewirken würde. Einige Versuche (s. Tabelle III) haben aber gezeigt, daß das Gegenteil der Fall ist und mit zerriebener Dauerhefe immer eine Verzögerung der Gärwirkung auftritt, die sich nach mehreren Tagen der Wirkung allerdings ausgleicht, so daß nach 96 Stunden der Gesamteffekt der zer-

1) Vergl. Hanow, Chem.-Zeitg., Cöthen, Bd. XXVIII, S. 684 (1904). 
riebenen Dauerhefe meistens der nämliche (oder im letzten Versuch sogar größer) ist, wie von dem unzerriebenen Präparate. Der Durchtritt des gelösten Rohrzuckers durch die Zellmembran der Acetondauerhefe findet demnach so rasch statt, daß damit keine merkbare Verzögerung der Gärwirkung verbunden ist.

$$
\text { Tabelle III. }
$$

Gärkraft von zerriebener Acetondauerhefe. Je $2 \mathrm{~g}$ Dauerhefe $+4 \mathrm{~g}$ Rohrzucker $+10 \mathrm{ccm}$ Wasser $+0,2 \mathrm{ccm}$ Toluol. $22^{\circ}$.

\begin{tabular}{|c|c|c|c|c|c|c|}
\hline \multirow{2}{*}{ Datum } & \multirow{2}{*}{$\begin{array}{c}\text { Nummer } \\
\text { des } \\
\text { Versuches }\end{array}$} & \multirow{2}{*}{$\begin{array}{c}\text { Acetondauerhefe } \\
\text { je } 2 \mathrm{~g}\end{array}$} & \multicolumn{4}{|c|}{$\begin{array}{c}\text { Kohlendioxyd in Grammen } \\
\text { nach Stunden }\end{array}$} \\
\hline & & & 24 & 48 & 72 & 96 \\
\hline \multirow[t]{4}{*}{ April 1903} & 1 & nicht zerrieben & 0,45 & 0,78 & 0,85 & 0,85 \\
\hline & 2 & $\gg$ & 0,46 & 0,78 & 0,85 & 0,85 \\
\hline & 3 & zerrieben & 0,34 & 0,65 & 0,80 & 0,85 \\
\hline & 4 & > & 0,34 & 0,65 & 0,82 & 0,88 \\
\hline \multirow[t]{4}{*}{ Novemb. 1903} & 1 & nicht zerrieben & 0,42 & 0,63 & 0,66 & 0,68 \\
\hline & 2 & 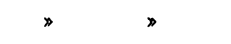 & 0,43 & 0,65 & 0,68 & 0,69 \\
\hline & 3 & zerrieben & 0,33 & 0,56 & 0,67 & 0,70 \\
\hline & 4 & » & 0,33 & 0,57 & 0,68 & 0,70 \\
\hline \multirow[t]{4}{*}{ 3. März 1905} & 1 & nicht zerrieben & 0,29 & 0,45 & 0,61 & 0,69 \\
\hline & 2 & $>\quad>$ & 0,28 & 0,46 & 0,61 & 0,69 \\
\hline & 3 & zerrieben & 0,26 & 0,49 & 0,69 & 0,78 \\
\hline & 4 & » & 0,26 & 0,46 & 0,66 & 0,75 \\
\hline
\end{tabular}

Die Zerreibung hatte bei diesen Versuchen in einer Reibschale mit der Hand stattgefunden und gemäß der mikroskopischen Untersuchung bei halbstündiger Dauer zu vollständiger Zertrümmerung von etwa einem Drittel der Zellen geführt. Da 
bei dieser Anordnung während des Zerreibens infolge von kräftigem Aufdrücken des Pistilles mit der Hand örtliche Überhitzung des Präparates zu befürchten war, wie denn tatsächlich beim Zerreiben von Sand in einer Porzellanschale unter Umständen ein brandiger Geruch auftritt, ähnlich dem bei Anschlagen von Feuerstein, wurde bei zwei weiteren Versuchen (s. Tabelle IV) die Pulverisierung mit einer kleinen Zerreibungsmaschine $^{1}$ ) vorgenommen. In einer horizontal rotierenden Porzellanschale (150 Touren in der Minute) wurden je $4 \mathrm{~g}$ Dauerhefe durch einen geradlinig hin- und hergehenden Stößel von $1750 \mathrm{~g}$ Gewicht zerkleinert. Bei der Unterbrechung nach 45 Minuten ergab die Prüfung unter dem Mikroskop, daß schätzungsweise die Hälfte der Zellen vollständig zertrümmert war.

Ferner tauchte die Vermutung auf, daß die Schädigung der Zymase in der zerriebenen Hefe am Anfang der Versuche vielleicht auf direkte Berührung mit dem Wasser bezw. der Zuckerlösung beruht, während dieses Enzym bei der unverletzten Dauerhefe unter dem Schutze der Zellmembran im Innern der Zellen in einer verhältnismäßig konzentrierten Lösung von Kolloidstoffen besser zu wirken vermag. Kolloidal gelöste Stoffe, auch solche anorganischer Natur, scheinen ganz allgemein durch die Gegenwart anderer Kolloidstoffe vor dem Ausflocken bewahrt zu werden. ${ }^{2}$ ) Wir müssen in dem Aufbau der höheren Organismen in Zellen eine Art von Schutzvorrichtung für die Beständigkeit kolloidaler Lösungen in denselben erblicken. In Berücksichtigung dieser Umstände wurde nun zerriebene und unzerriebene Dauerhefe in frischen Hefepreßsaft eingetragen und unter Zuckerzusatz die Gärkraft bestimmt. Aber obwohl auf diesem Wege auch für die zerriebene Dauerhefe der nötige Schutz durch Kolloidstoffe gewährleistet war, haben wir wiederum regelmäßig eine geringere Wirkung des gepulverten Präparates innerhalb der ersten 24 Stunden erhalten, die sich bei mehrtägiger Versuchsdauer allerdings

1) Siehe ‘Die Zymasegärung`, S. 61 (1903).

8) Vergl. G. Pa al, Ber. d. Deutsch. chem. Ges., Bd. XXXV, S. 2209 (1902), woselbst auch weitere Literaturangaben. Siehe ferner J. Meisenheimer, Diese Zeitschrift, Bd. XXXVII, S. 521 (1903). 
dann ausglich. Es tritt also durch das Zerreiben der Dauerhefe eine meistens vorübergehende Schädigung der Gärkraft ein, gleichgültig ob die Dauerhefe nur mit reiner Zuckerlösung oder mit gezuckertem Hefepreßsaft, also einer Kolloidlösung, übergossen wird.

Die spezielle Anordnung der zwei Versuchsreihen der Tabelle IV war folgende: Zunächst wurde die Gärkraft des verwendeten Preßsaftes (Nr. 1 und 2) und der verwendeten Acetondauerhefe in unzerriebenem (Nr. 3 und 4), sowie in zerriebenem Zustande (Nr. 5 und 6) bestimmt, endlich die Gärkraft der nämlichen Dauerhefe nach Eintragen in Preßsaft, ohne (Nr. 7 und 8) und nach Zerreibung des Präparates (Nr. 9 und 10). In allen Fällen wurden zwei Parallelversuche ausgeführt. Es empfahl sich, jedesmal nur $10 \mathrm{ccm}$ Preßsaft und $1 \mathrm{~g}$ Dauerhefe zu benutzen, da bei der vereinigten Wirkung beider sonst, wie Vorversuche lehrten, infolge zu lebhafter Blasenbildung leicht Überschäumen eintritt. Um selbst im Falle Übersteigens geringer Schaummengen die Versuche zu Ende führen zu können, wurden bei Nummer 7-10 an die Versuchskölbchen noch leere Kölbchen angeschlossen, diese erst mit dem Schwefelsäuregärverschluß versehen und mitgewogen. Nach Beendigung der Versuche wurde durch alle Kölbchen noch getrocknete Luft geleitet, um das angesammelte Kohlendioxyd $z u$ entfernen.

\section{Einwirkung verschiedener Stoffe auf die Gärkraft des Hefepreßsaftes.}

Im Anschluß an frühere ähnliche Versuche soll im folgenden über die Einwirkung von Formaldehyd, Natriumfluorid, Chininchlorhydrat, Alkohol und Aceton berichtet werden.

\section{Formaldehyd}

unterdrückt schon bei Zusätzen von $0,04 \%$ die Gärung durch Hefe. ${ }^{1)}$ Es schien deshalb möglich, daß geeignete Konzentrationen dieses Stoffes, welche das Wachstum und die Gärtätigkeit der Hefe bereits verhindern, für die Zymase nur wenig Schädigung mit sich bringen.

1) Cluß und Felber, Zeitschrift für Spiritus-Industrie, Bd. XXI, S. 29 (1898); Flügge, Mikroorganismen, Bd. I, S. 464. 
Weitere Versuche über die zellfreie Gärung.

Tabelle IV.

Gärkraft von zerriebener Acetondauerhefe bei Zusatz von Hefepreßsaft.

Je $10 \mathrm{ccm}$ Preßsaft (bezw. $1 \mathrm{~g}$ Dauerhefe, zerrieben oder unzerrieben, $\left.+10 \mathrm{ccm} \mathrm{H}_{2} \mathrm{O}\right)+4 \mathrm{~g}$ Rohrzucker $+0,1 \mathrm{ccm}$ Toluol. $22^{\circ}$.

\begin{tabular}{|c|c|c|c|c|c|c|c|c|c|c|}
\hline \multirow{3}{*}{ Datum } & \multirow{3}{*}{$\begin{array}{c}\text { Nr. } \\
\text { des } \\
\text { Ver- } \\
\text { suches }\end{array}$} & \multirow{3}{*}{$\begin{array}{c}\text { Preß- } \\
\text { saft } \\
\text { ccm }\end{array}$} & \multirow{3}{*}{$\begin{array}{l}\text { Wasser } \\
\mathrm{ccm}\end{array}$} & \multicolumn{2}{|c|}{$\begin{array}{c}\text { Dauerhefe } \\
\text { in } \mathrm{g}\end{array}$} & \multicolumn{5}{|c|}{$\begin{array}{c}\text { Kohlendioxyd in Grammen } \\
\text { nach Stunden }\end{array}$} \\
\hline & & & & \multirow{2}{*}{$\begin{array}{c}\text { zer- } \\
\text { rieben }\end{array}$} & \multirow{2}{*}{\begin{tabular}{|c|} 
nicht \\
zer- \\
rieben
\end{tabular}} & \multirow[t]{2}{*}{24} & \multirow[t]{2}{*}{48} & \multirow[t]{2}{*}{72} & \multicolumn{2}{|c|}{$\begin{array}{c}96 \\
\text { Luftdurch- } \\
\text { leitung }\end{array}$} \\
\hline & & & & & & & & & ohne & mit \\
\hline \multirow[t]{10}{*}{ 10. II. $0 \check{\jmath}$} & 1 & 10 & 0 & 0 & 0 & 0,47 & 0,68 & 0,73 & 0,74 & 0,78 \\
\hline & 2 & 10 & 0 & 0 & 0 & 0,47 & 0,68 & 0,73 & 0,74 & 0,78 \\
\hline & 3 & 0 & 10 & 0 & 1 & 0,19 & 0,33 & 0,41 & 0,44 & 0,48 \\
\hline & 4 & 0 & 10 & 0 & 1 & 0,20 & 0,32 & 0,41 & 0,44 & 0,48 \\
\hline & 5 & 0 & 10 & 1 & 0 & 0,10 & 0,19 & 0,25 & 0,29 & $0,33$. \\
\hline & 6 & 0 & 10 & 1 & 0 & 0,11 & 0,21 & 0,27 & 0,32 & 0,36 \\
\hline & 7 & 10 & 0 & 0 & 1 & 0,81 & 1,01 & 1,05 & 1,06 & 1,14 \\
\hline & 8 & 10 & 0 & 0 & 1 & 0,80 & 1,00 & 1,03 & 1,04 & 1,12 \\
\hline & 9 & 10 & 0 & 1 & 0 & 0,72 & 0,99 & $\mid 1,06$ & 1,07 & 1,15 \\
\hline & 10 & 10 & 0 & 1 & 0 & 0,73 & 1,01 & 1,07 & 1,09 & 1,17 \\
\hline \multirow[t]{10}{*}{ 13. II. 05} & 1 & 10 & 0 & 0 & 0 & 0,43 & 0,69 & 0,76 & 0,77 & 0,81 \\
\hline & 2 & 10 & 0 & 0 & 0 & 0,42 & 0,68 & 0,76 & 0,78 & 0,82 \\
\hline & 3 & 0 & 10 & 0 & 1 & 0,15 & 0,30 & 0,38 & 0,43 & 0,47 \\
\hline & 4 & 0 & 10 & 0 & 1 & 0,18 & 0,33 & 0,43 & 0,46 & 0,50 \\
\hline & 5 & 0 & 10 & 1 & 0 & 0,13 & 0,26 & 0,34 & 0,40 & 0,44 \\
\hline & 6 & 0 & 10 & 1 & 0 & 0,13 & 0,25 & 0,35 & 0,41 & 0,45 \\
\hline & 7 & 10 & 0 & 0 & 1 & 0,74 & 1,01 & 1,05 & 1,05 & 1,13 \\
\hline & 8 & 10 & 0 & 0 & 1 & 0,73 & 0,98 & 1,01 & 1,03 & 1,11 \\
\hline & 9 & 10 & 0 & 1 & 0 & 0,68 & 0,99 & 1,03 & 1,04 & 1,12 \\
\hline & 10 & 10 & 0 & 1 & 0 & 0,70 & 1,02 & 1,02 & 1,02 & 1,10 \\
\hline
\end{tabular}


Über die Einwirkung dieses Antiseptikums auf gärenden Preßsaft liegen bis jetzt nur ${ }^{1}$ ) einige Angaben von A. Wróblews$\mathrm{ki}^{2}$ ) vor, wonach Zusatz von $0,05 \%$ Formaldehyd die Gärwirkung des Saftes auf $1_{12}$, von $0,25 \%$ Formaldehyd ungefähr auf $1 / 100$ herabdrückt. Unsere Versuche ergaben, daß diese Zahlen, die mit Preßsaft von sehr geringer Gärwirkung ermittelt sind, für einigermaßen gärkräftigen Preßsaft nicht zutreffen. Wir haben bei einer Reihe von Parallelversuchen 0,12 bezw. 0,18 bezw. 0,24\% Formaldehyd (berechnet auf die Menge des Saftes) zugegeben und dadurch schrittweise Abnahme der gesamten Gärkraft bewirkt, die aber durch $0,12 \%$ nur $1 / 5$, durch $0,24 \% 1 / 3 \ldots 3 / 5$ betrug. Immerhin entwickelten auch bei Zusatz von 0,24\% Formaldehyd, einer Menge, die das Wachstum von lebenden Organismen sicher ausschließt, umsomehr, als außerdem noch 1\% Toluol zugesetzt war, $20 \mathrm{ccm}$ Saft $0,3-0,5 \mathrm{~g} \mathrm{CO}_{2}$.

Formaldehyd hat demnach auf empfindliche Enzyme keine sehr starke Einwirkung und dürfte in manchen Fällen als brauchbares Antiseptikum Verwendung finden können. In der Tat haben z. B. Versuche von M. Delbrück und F. Rothenbach ${ }^{3}$ ) auch gezeigt, daß diese Substanz ein geeignetes Brennereiantiseptikum behufs Unterdrückung von Spaltpilzen darstellt.

1) Die wenigen Versuche von A. Macfadyen, Morris und Rowland zu dieser Frage (s. Ber. d. D. chem. Ges., Bd. XXXIII, S. 2782 (1900)) sind, weil mit schlecht wirkendem Preßsaft und wahrscheinlich bei Gegenwart von Bakterien ausgeführt, wie der eine von uns nachweisen konnte (Buchner, ebenda XXXIII, S. 3311 (1900)), so wenig zuverlässig, daß sie am besten unberücksichtigt bleiben. Macfadyen hat sich dieser Kritik übrigens später selbst angeschlossen (Verhandl. Ges. d. Naturforscher und Ärzte, 1904, Bd. I, S. 235) und Harden und Young kamen bei direkter Nachprüfung einer Reihe von Versuchen der genannten Forscher auch zu vollständig anderen Resultaten (Ber. d. D. chem. Ges., Bd. XXXVII, S. 1052 (1904)).

2) Journ. f. prakt. Chem. (2), Bd. LXIV, S. 17 (1901).

s) Maercker-Delbrück, Spiritusfabrikation, Berlin 1903, S. 667. 
Weitere Versuche über die zellfreie Gärung.

Tabelle V.

Gärwirkung des Preßsaftes bei Zusatz von Formaldehyd.

Je $20 \mathrm{ccm}$ Saft $+1,2 \mathrm{ccm}$ Formaldehydlösung (von $4 \%$ ) bezw. Wasser $+8 \mathrm{~g}$ Rohrzucker $+0,2 \mathrm{ccm}$ Toluol. $22^{\circ}$.

\begin{tabular}{|c|c|c|c|c|c|c|c|}
\hline \multirow[b]{2}{*}{ Datum } & \multicolumn{3}{|c|}{ Zusatz von } & \multicolumn{4}{|c|}{$\begin{array}{c}\text { Kohlendioxyd in Grammen } \\
\text { nach Stunden }\end{array}$} \\
\hline & $\begin{array}{l}\text { For } \\
\%\end{array}$ & $\begin{array}{c}\text { dehyd } \\
4 \% \% \text { ige } \\
\text { Lösung } \\
\text { ccm }\end{array}$ & $\begin{array}{c}\text { Wasser } \\
\text { ccm }\end{array}$ & 24 & 48 & 72 & 96 \\
\hline \multirow[t]{3}{*}{ 28. X. 04} & $\begin{array}{l}0,12 \\
0,12\end{array}$ & $\begin{array}{l}0,6 \\
0,6\end{array}$ & $\begin{array}{l}0,6 \\
0,6\end{array}$ & $\begin{array}{l}0,26 \\
0,23\end{array}$ & - & $\begin{array}{l}0,52 \\
0,51\end{array}$ & $\begin{array}{l}0,53 \\
0,53\end{array}$ \\
\hline & $\begin{array}{l}0,18 \\
0,18\end{array}$ & $\begin{array}{l}0,9 \\
0,9\end{array}$ & $\begin{array}{l}0,3 \\
0,3\end{array}$ & $\begin{array}{l}0,21 \\
0,21\end{array}$ & - & $\begin{array}{l}0,44 \\
0,43\end{array}$ & $\begin{array}{l}0,45 \\
0,44\end{array}$ \\
\hline & $\begin{array}{l}0,24 \\
0,24\end{array}$ & $\begin{array}{l}1,2 \\
1,2\end{array}$ & $\begin{array}{l}0 \\
0\end{array}$ & $\begin{array}{l}0,16 \\
0,16\end{array}$ & - & $\begin{array}{l}0,36 \\
0,35\end{array}$ & $\begin{array}{l}0,37 \\
0,36\end{array}$ \\
\hline \multirow[t]{4}{*}{ 4. XI. 04} & $\begin{array}{l}0 \\
0\end{array}$ & $\begin{array}{l}0 \\
0\end{array}$ & $\begin{array}{l}0 \\
0\end{array}$ & - & $\begin{array}{l}0,40 \\
0,39\end{array}$ & $\begin{array}{l}0,40 \\
0,40\end{array}$ & $\begin{array}{l}0, \mathbb{4 1} \\
0, \mathbb{4 1}\end{array}$ \\
\hline & $\begin{array}{l}0,12 \\
0,12\end{array}$ & $\begin{array}{l}0,6 \\
0,6\end{array}$ & $\begin{array}{l}0,6 \\
0,6\end{array}$ & - & $\begin{array}{l}0,31 \\
0,32\end{array}$ & $\begin{array}{l}0,33 \\
0,33\end{array}$ & $\begin{array}{l}0,35 \\
0,33\end{array}$ \\
\hline & $\begin{array}{l}0,18 \\
0,18\end{array}$ & $\begin{array}{l}0,9 \\
0,9\end{array}$ & $\begin{array}{l}0,3 \\
0,3\end{array}$ & - & $\begin{array}{l}0,28 \\
0,27\end{array}$ & $\begin{array}{l}0,30 \\
0,29\end{array}$ & $\begin{array}{l}0,30 \\
0,30\end{array}$ \\
\hline & $\begin{array}{l}0,24 \\
0,24\end{array}$ & $\begin{array}{l}1,2 \\
1,2\end{array}$ & $\begin{array}{l}0 \\
0\end{array}$ & - & $\begin{array}{l}0,24 \\
0,24\end{array}$ & $\begin{array}{l}0,27 \\
0,26\end{array}$ & $\begin{array}{l}0,30 \\
0,27\end{array}$ \\
\hline \multirow[t]{4}{*}{ 22. XI. 04} & $\begin{array}{l}0 \\
0\end{array}$ & $\begin{array}{l}0 \\
0\end{array}$ & $\begin{array}{l}1,2 \\
1,2\end{array}$ & $\begin{array}{l}0,91 \\
0,91\end{array}$ & $\begin{array}{l}1,08 \\
1,07\end{array}$ & $\begin{array}{l}1,12 \\
1,10\end{array}$ & - \\
\hline & $\begin{array}{l}0,12 \\
0,12\end{array}$ & $\begin{array}{l}0,6 \\
0,6\end{array}$ & $\begin{array}{l}0,6 \\
0,6\end{array}$ & $\begin{array}{l}0,77 \\
0,74\end{array}$ & $\begin{array}{l}0,89 \\
0,87\end{array}$ & $\begin{array}{l}0,92 \\
0,90\end{array}$ & - \\
\hline & $\begin{array}{l}0,18 \\
0,18\end{array}$ & $\begin{array}{l}0,9 \\
0,9\end{array}$ & $\begin{array}{l}0,3 \\
0,3\end{array}$ & $\begin{array}{l}0,58 \\
0,60\end{array}$ & $\begin{array}{l}0,67 \\
0,73\end{array}$ & $\begin{array}{l}0,71 \\
0,75\end{array}$ & - \\
\hline & $\begin{array}{l}0,24 \\
0,24\end{array}$ & $\begin{array}{l}1,2 \\
1,2\end{array}$ & $\begin{array}{l}0 \\
0\end{array}$ & $\begin{array}{l}0,39 \\
0,38\end{array}$ & $\begin{array}{l}0,47 \\
0,45\end{array}$ & $\begin{array}{l}0,50 \\
0,49\end{array}$ & - \\
\hline
\end{tabular}


Tabelle VI.

Gärwirkung des Preßsaftes bei Zusatz von Natriumfluorid.

Je $20 \mathrm{ccm}$ Saft $+8 \mathrm{~g}$ Rohrzucker $+0,2 \mathrm{ccm}$ Toluol. $22^{\circ}$.

\begin{tabular}{|c|c|c|c|c|c|}
\hline \multirow[t]{2}{*}{ Datum } & \multicolumn{2}{|c|}{$\begin{array}{c}\text { Zusatz von } \\
\text { Natriumfluorid }\end{array}$} & \multicolumn{3}{|c|}{$\begin{array}{c}\text { Kohlendioxyd in Grammen } \\
\text { nach Stunden }\end{array}$} \\
\hline & $\%$ & g & 24 & 48 & 72 \\
\hline \multirow[t]{6}{*}{ 31. I. 05} & 0 & 0 & 1,37 & 1,74 & - \\
\hline & 0 & 0 & 1,33 & 1,75 & - \\
\hline & 0,5 & 0,1 & 0,03 & 0,04 & - \\
\hline & 0,5 & 0,1 & 0,02 & 0,04 & - \\
\hline & 1,0 & 0,2 & 0,03 & 0,09 & - \\
\hline & 2,0 & 0,4 & 0,02 & 0,04 & - \\
\hline \multirow[t]{8}{*}{ 7. II. 05} & 0 & 0 & 0,94 & 1,19 & 1,27 \\
\hline & 0 & 0 & 1,00 & 1,27 & 1,30 \\
\hline & 0,5 & 0,1 & 0,05 & 0,11 & 0,15 \\
\hline & 0,5 & 0,1 & 0,05 & 0,11 & 0,16 \\
\hline & 1,0 & 0,2 & 0,02 & 0,04 & 0,04 \\
\hline & 1,0 & 0,2 & 0,01 & 0,01 & 0,01 \\
\hline & 2,0 & 0,4 & 0,02 & 0,02 & 0,02 \\
\hline & 2,0 & 0,4 & 0,02 & 0,02 & 0,02 \\
\hline
\end{tabular}

Natriumfluorid

in einer Konzentration von $1-2 \%$ schützt gegen Bakterienwachstum. ${ }^{1}$ ) Über die Einwirkung von Fluoriden auf Zuckergärung durch Hefepreßsaft lagen bisher nur einige qualitative Versuche mit Ammoniumfluorid ${ }^{2}$ ) vor, welche eine hemmende Wirkung von Mengen von $0,5-2 \%$ ergaben. In Tab. VI 633 (1904).

1) Vergl. z. B. Portier, Ann. de l'inst. Pasteur, Bd. XVIII, S.

2) E. u. H. Buchner u. M. Hahn, Die Zymasegärung, S. 184. 
sind nun quantitative Versuche mit Zusatz von Natriumfluorid (von C. A. F. Kahlbaum bezogen, löst sich in Wasser mit stark alkalischer Reaktion) in ähnlichen Konzentrationen mitgeteilt, welche die außerordentlich große Schädlichkeit dieses Salzes für die Wirkung der Zymase bestätigen. Dieser Sachverhalt muß auffallend erscheinen, weil nach dem Verfahren von Effront bei der Vergärung der Maische in dem Brennereibetrieb häufig Fluoride zugesetzt werden. ${ }^{1}$ ) Allerdings ist die dabei angewandte Menge, z. B. 0,03\% Aluminiumfluorid, eine beträchtlich geringere und Zellmembran nebst Plasmaschlauch schützen wohl die Zymase in den lebenden Zellen vor zu starker Einwirkung der Antiseptika. Da sich bei unserer ersten Versuchsreihe das Natriumfluorid nur langsam im Preßsaft auflöste, wurde es für die zweite Serie vorher besonders sorgfältig gepulvert.

\section{Chininchlorhydrat.}

Nach Angaben von Frl. 0. Grigoriew ${ }^{2}$ ) erhöht Zusatz von salzsaurem Chinin die Einwirkung von Acetondauerhefe auf Rohrzuckerlösung. Diese interessanten Versuche geben jedoch infolge ihrer Anordnung keinen Aufschluß über die Gesamtmengen von Kohlendioxyd, die mit oder ohne Zusatz des Salzes entwickelt werden, sondern nur über den Verlauf des Gärungsvorganges in einzelnen Zeiträumen. Wir haben sie deshalb mit Hefepreßsaft unter Anwendung unseres gewöhnlichen Verfahrens unter Bestimmung des Kohlensäureanhydrids durch Gewichtsverlust wiederholt und tatsächlich auch eine, wenngleich geringe, Verstärkung der Gärwirkung bei Zusatz von $0,05 \%$ Chininchlorhydrat (Ka hlbau m) beobachten können. Frl. T. Gromow ${ }^{3}$ ) hat ermittelt, daß es sich dabei um eine hemmende Wirkung des Chininsalzes auf den ungünstigen Einfluß des proteolytischen Enzymes für die Zymase handelt. Damit stimmt überein, daß nach unseren Versuchen die höheren

1) Vergl. Maercker-Delbrück, Spiritusfabrikation, Berlin 1903, S. 666 .

2) Diese Zeitschrift, Bd. XLII, S. 323 (1904).

s) Ebenda, XLII, S. 309 (1904). 
$\mathrm{CO}_{2}$-Zahlen bei Zusatz von $0,5 \%$ des Salzes auch erst nach längerer Gärwirkung in die Erscheinung treten. Bei Zusatz von $1 \%$ Chininchlorhydat ist das Maximum der günstigen Wirkung offenbar schon wieder überschritten. Da $0,18 \%$ Chininchlorhydrat das Wachstum des Staphylococcus pyogen. aur. behindern, $\left.{ }^{1}\right)$ dürfte ein $1 \%$ iger Zusatz dieses Mittels, zumal bei Gegenwart von Toluol, die Abwesenheit von allen Mikroorganismen sicher verbürgen. Die Versuche bilden demnach eine weitere Bestätigung, daß es sich nicht um die Wirkung von Mikroorganismen oder lebenden Protoplasmasplittern handelt.

Tabelle VII.

Gärwirkung von Acetondauerhefe hei Zusatz von Chininchlorhydrat.

Je $2 \mathrm{~g}$ Acetondauerhefe $+10 \mathrm{ccm} \mathrm{H}_{2} \mathrm{O}+4 \mathrm{~g}$ Rohrzucker $+0,2 \mathrm{ccm}$ Toluol. $22^{\circ}$.

\begin{tabular}{|c|c|c|c|c|c|c|c|c|}
\hline \multirow{2}{*}{ Datum } & \multicolumn{2}{|c|}{$\begin{array}{l}\text { Zusatz von Chinin- } \\
\text { chlorhydrat }\end{array}$} & \multicolumn{6}{|c|}{ Kohlendioxyd in $\mathrm{g}$ nach Stunden } \\
\hline & $\%$ & g & 24 & 48 & 72 & 96 & 120 & 144 \\
\hline \multirow[t]{6}{*}{ 22. XI. 04} & 0 & 0 & 0,34 & 0,70 & 0,88 & 0,94 & 0,97 & 1,00 \\
\hline & 0 & 0 & 0,34 & 0,69 & 0,85 & 0,90 & 0,90 & 0,91 \\
\hline & 0,5 & 0,05 & 0,35 & 0,71 & 0,93 & 1,07 & 1,11 & 1,13 \\
\hline & 0,5 & 0,05 & 0,35 & 0,70 & 0,92 & 1,06 & 1,09 & 1,12 \\
\hline & 1,0 & 0,1 & 0,33 & 0,64 & 0,83 & 0,99 & 1,04 & 1,06 \\
\hline & 1,0 & 0,1 & 0,32 & 0,62 & 0,81 & 0,97 & 1,02 & 1,04 \\
\hline \multirow[t]{6}{*}{ 10. II. 05} & 0 & 0 & 0,65 & 0,96 & 1,05 & 1,09 & 1,09 & 1,10 \\
\hline & 0 & 0 & 0,69 & 1,00 & 1,10 & 1,13 & 1,14 & 1,14 \\
\hline & 0,5 & 0,05 & 0,64 & 0,94 & 1,06 & 1,14 & 1,18 & 1,20 \\
\hline & 0,5 & 0,05 & 0,65 & 0,97 & 1,08 & 1,15 & 1,17 & 1,17 \\
\hline & 1,0 & 0,1 & 0,55 & 0,81 & 0,91 & 0,96 & 0,98 & 0,99 \\
\hline & 1,0 & 0,1 & 0,57 & 0,84 & 0,95 & 1,02 & 1,07 & 1,08 \\
\hline
\end{tabular}

1) Flügge, Mikroorganismen, Bd. I, S. 472; vgl. ferner Duclaux, Mikrobiologie, Bd. III, S. 492. 


\section{Zusatz von Äthylalkohol}

zu gärendem Preßsaft ist bisher nur in einigen wenigen Fällen versucht worden, welche im allgemeinen schädlichen Einfluß ergeben haben. ${ }^{1}$ ) Es liegen nunmehr umfangreichere Versuche vor (s. Tab. VIII a.f. S.), welche die schrittweise Abnahme der Gärkraft mit steigendem Alkoholzusatz erkennen lassen. Diese betrug bei Zusatz von $6 \%$ Alkohol bei einem Versuch $1 / 5$ der Gesamtgärleistung, bei zwei weiteren noch weniger. Das Zusetzen des Alkohols wird durch Eintropfen in den fortwährend bewegten Saft bewerkstelligt, wobei sogleich geringe Trübungen auftreten, die im Verlauf der Gärung sehr bedeutenden Umfang annehmen. Eine Begünstigung der Zymasewirkung durch Zusatz von 5\% Äthylalkohol, wie sie Frl. Grigoriew ${ }^{2}$ ) beobachtet und auf Hemmung der schädlichen Endotryptasewirkung zurückgeführt hat, konnten wir nicht nachweisen. Bei Zusatz von 10 bezw. 14\% Alkohol geht die Gärwirkung außerordentlich, nämlich auf ungefähr $1 / 2$ bezw. ${ }^{1 / 4}-1 / 5$, herab, bleibt aber immerhin noch deutlich nachweisbar. Bei der Gärung durch lebende Hefe steigt der Alkoholgehalt im Höchstfalle auf $14-15 \%,{ }^{3}$ ) worauf die Einwirkung auf den Zucker aufhört.

\section{Aceton.}

Bei der Darstellung von Dauerpräparaten durch Eintragen abgepreßter Hefe in Alkohol und Äther hat sich der Ersatz des Alkohols durch Aceton sehr bewährt, indem es auf diesem von R. Rapp aufgefundenen Wege leichter gelingt, stark gärkräftige Dauerhefe zu erzielen. ${ }^{4}$ ) Es schien deshalb wahrscheinlich, daß Acetonzusatz die Gärwirkung des Preßsaftes weniger schädigt, als der Zusatz von Alkohol, eine Vermutung, die sich aber, wie Tab. IX zeigt, nicht bestätigt hat. Im Gegenteil wirkt (bei den angewandten Mengen) Aceton, das im Preßsaft ähnliche Trübungen hervorruft, wie der Alkohol, schädlicher als letzterer.

1) «Zymasegärung», S. 174 (München 1903). S. ferner A. Wróblewski, Journ. f. prakt. Chem., Bd. LXIV, S. 22 (1901).

2) Diese Zeitschrift, Bd. XLII, S. 323 (1904).

3) Vergl. E. Duclaux, Mikrobiologie, Bd. I, S. 239.

4) «Zymasegärung», S. 265 (1903).

Hoppe-Seyler's Zeitschrift f. physiol. Chemie. XLIV. 


\section{Tabelle VIII.}

Gärwirkung des Preßsaftes bei Zusatz von Äthylalkohol. ${ }^{1}$ )

Je $20 \mathrm{ccm}$ Preßsaft $+8 \mathrm{~g}$ Rohrzucker $+0,2 \mathrm{ccm}$ Toluol. $22^{\circ}$.

\begin{tabular}{|c|c|c|c|c|c|c|}
\hline \multirow[t]{2}{*}{ Datum } & \multicolumn{2}{|c|}{$\begin{array}{c}\text { Zusatz } \\
\text { von Alkohol }\end{array}$} & \multicolumn{4}{|c|}{$\begin{array}{c}\text { Kohlendioxyd } \\
\text { in Grammen nach Sturden }\end{array}$} \\
\hline & $\%$ & $\mathrm{ccm}$ & 24 & 48 & 72 & 96 \\
\hline \multirow[t]{6}{*}{ 3. XII. 04} & 0 & 0 & - & 0,50 & 0,52 & - \\
\hline & 0,5 & 0,1 & - & 0,48 & 0,50 & - \\
\hline & 1,0 & 0,2 & - & 0,48 & 0,50 & - \\
\hline & 2,0 & 0,4 & - & 0,49 & 0,50 & - \\
\hline & 4,0 & 0,8 & - & 0,49 & 0,51 & - \\
\hline & 6,0 & 1,2 & - & 0,47 & 0,49 & - \\
\hline \multirow[t]{6}{*}{ 8. XII. 04} & 0 & 0 & 0,54 & 0,77 & - & 0,82 \\
\hline & 0,5 & 0,1 & 0,56 & 0,76 & - & 0,81 \\
\hline & 1,0 & 0,2 & 0,52 & 0,70 & - & 0,77 \\
\hline & 2,0 & 0,4 & 0,55 & 0,72 & - & 0,75 \\
\hline & 4,0 & 0,8 & 0,50 & 0,67 & - & 0,71 \\
\hline & 6,0 & 1,2 & 0,50 & 0,67 & - & 0,70 \\
\hline \multirow[t]{3}{*}{ 10. I. 05} & 0 & 0 & 0,68 & 0,94 & 1,05 & 1,07 \\
\hline & 2,0 & 0,4 & 0,67 & 0,88 & 0,99 & 1,00 \\
\hline & 6,0 & 1,2 & 0,58 & 0,78 & 0,85 & 0,87 \\
\hline \multirow[t]{3}{*}{ 9. I. 05} & 0 & 0 & 0,61 & 0,81 & 0,87 & - \\
\hline & 10 & 2,0 & 0,26 & 0,35 & 0,41 & - \\
\hline & 14 & 2,8 & 0,13 & 0,17 & 0,17 & - \\
\hline \multirow[t]{3}{*}{ 7. II. 05} & 0 & 0 & 0,97 & 1,22 & 1,28 & - \\
\hline & 10 & 2,0 & 0,49 & 0,63 & 0,66 & - \\
\hline & 14 & 2,8 & 0,29 & 0,35 & 0,36 & - \\
\hline
\end{tabular}

1) Die angegebenen Zahlen stellen das Mittel von je zwei Parallelversuchen dar, zwischen welchen die Maximalschwankung nicht mehr als 0,04 betrug. 
Tabelle IX.

Gärwirkung des Preßsaftes bei Zusatz von Aceton. ${ }^{1}$ ) Je $20 \mathrm{ccm}$ Preßsaft $+8 \mathrm{~g}$ Rohrzucker $+0,2 \mathrm{ccm}$ Toluol. $22^{\circ}$.

\begin{tabular}{c|c|c|c|c|c|c}
\hline \hline \multirow{2}{*}{ Datum } & \multicolumn{2}{|c|}{$\begin{array}{c}\text { Zusatz } \\
\text { von Aceton }\end{array}$} & \multicolumn{4}{|c}{$\begin{array}{c}\text { Kohlendioxyd } \\
\text { in Grammen nach Stunden }\end{array}$} \\
\cline { 2 - 7 } & $\%$ & $\mathrm{ccm}$ & 24 & 48 & 72 & 96 \\
\hline \multirow{2}{*}{ 10. I. 05 } & 0 & 0 & 0,68 & 0,94 & 1,05 & 1,07 \\
& 2,0 & 0,4 & 0,64 & 0,85 & 0,93 & 0,95 \\
& 6,0 & 1,2 & 0,54 & 0,71 & 0,78 & 0,80 \\
\hline \multirow{2}{*}{9. I. 05 } & 0 & 0 & 0,61 & 0,81 & 0,87 & - \\
& 10,0 & 2,0 & 0,23 & 0,30 & 0,34 & - \\
& 14,0 & 2,8 & 0,12 & 0,13 & 0,13 & -
\end{tabular}

\section{Anhang:}

E. Buchner, Entgegnung an Herrn Hugo Fischer.

Verschiedene von Hugo Fischer in Bonn über Enzyme und Protoplasma vor einiger Zeit geäußerte Ansichten ${ }^{2}$ ) machen meinen Widerspruch notwendig. Dieser Autor betrachtet noch immer ganz allgemein die Enzyme als «lebend», eine Anschauung, welche unter den jetzigen Naturforschern glücklicherweise nur mehr vereinzelte Vertreter finden dürfte. ${ }^{3}$ ) Die Enzyme sind wahrscheinlich bestimmte chemische Stoffe (sog. chemische Verbindungen); es spricht wenigstens keine bisher erkannte Tatsache gegen eine solche Auffassung. Auch H. Fischer äußert die Meinung, daß "die Zymase vielleicht ein einheit-

1) Die Zahlen sind Durchschnittswerte von je zwei Parallelversuchen.

2) Zentralblatt f. Bakt., II. Abt., Bd. X, S. 452 (1903).

3) Solches läßt sich aus dem Verhalten der Herausgeber von Lehrbüchern $z u$ dieser Frage leicht beweisen. Es nennt z. B. Alfred Fischer in seinen Vorlesungen über Bakterien (2. Aufl., Jena 1903, S. 206, 207) das Enzym klar und deutlich seinen chemischen, vom lebenden Organismus erzeugten Körper , und strotz mancher an lebende Wesen erinnernden Eigenschaften ein lebloses Produkt». 
licher Körper sein könnte. « ${ }^{1}$ ) Wenn nun die Enzyme nach dem gleichen Autor als lebend betrachtet werden sollen, müßte es lebende chemische Moleküle geben. "Lebend» und chemisch aktiv wären dann identische Begriffe; labile Körper wie Ozon, Aldehyde, Kaliumpermanganat könnten als lebend aufgefaßt werden. Es gibt aber außer chemischen Verbindungen und abgesehen von den Elementen noch eine dritte Klasse von Körpern, was H. Fischer anscheinend übersieht, ${ }^{2}$ ) nämlich die Gemenge, welche aus verschiedenen chemischen Stoffen zusammengesetzt sind. «Lebend» können nur Gemenge sein, denn nur durch die Wechselbeziehungen zwischen den verschiedenen chemischen Stoffen kommen jene Erscheinungen zustande, deren Gesamtheit man als Lebensvorgänge bezeichnet.

Die übrigen kleinen Angriffe des Herrn H. Fischer sind rein dialektischer Art; ein Eingehen darauf halte ich nicht für nötig.

1) a. a. 0., S. 456 .

2) a. a. 0., S. 456 oben. 